\title{
AN EFFICIENT ALGORITHM FOR AUTOMATIC TUMOR DETECTION IN CONTRAST ENHANCED BREAST MRI BY USING ARTIFICIAL NEURAL NETWORK (NEUBREA)
}

\author{
Bulent Bayram*, Hilmi K. Koca ${ }^{\dagger}$ Burcu Narin $\ddagger$ G. Cigdem Cavdaroglu \\ Levent Celik\$ Ugur Acar, Rahmi Cubuk
}

\begin{abstract}
The advances in image processing technology contribute to the interpretation of medical images and early diagnosis. Moreover various studies can be found in medical journals dedicated to Artificial Neural Networks (ANN). In the presented study, a method was developed to learn and detect benign and malignant tumor types in contrast-enhanced breast magnetic resonance images (MRI). The backpropagation algorithm was taken as the ANN learning algorithm. The algorithm (NEUBREA) was developed in C\# programming language by using Fast Artificial Neural Network Library (FANN).

Having been diagnosed by radiologists, 7 cases of malignant tumor, 8 cases of benign tumor, and 3 normal cases were used as a training set. The results were tested on 34 cases that had been diagnosed by radiologists. After the comparison of the results, the overall accuracy of algorithm was defined as $92 \%$.
\end{abstract}

Key words: Computer aided detection, medical image processing, breast cancer, $A N N$

Received: October 27, 2010

Revised and accepted: August 19, 2013

*Bulent Bayram - Corresponding Author, Ugur Acar - Research Assist

Yildiz Technical University, Dept. of Geomatic, Davutpasa Campus, Davutpasa Str., 34210 Esenler/Istanbul-Turkey, E-mail: bulentbayram65@gmail.com, bayram@yildiz.edu.tr, uguracara@gmail.com, Tel.: 00902123835329 , Mob.: 00905327423759

${ }^{\dagger}$ Hilmi K. Koca, G. Cigdem Cavdaroglu - Engineer

Yildiz Technical University, Intitute of Science and Technology, Yildiz, 34349, Besiktas-Istanbul-

Turkey, E-mail: hkkoca@gmail.com, cavdarkizi@gmail.com

$\ddagger$ Burcu Narin - Radiologist

Haydarpasa Education and Research Hospital, Dept. of Radiology, Tibbiye Str. 34668, ÜsküdarIstanbul-Turkey, E-mail: burcu_narin@yahoo.com

$\S$ Levent Celik, Rahmi Çubuk - Radiologist

Maltepe University, 2006 Marmara Education Town, 34857 Maltepe-Istanbul/Turkey, E-mail:

leventcelik@hotmail.com, rahmicubuk@yahoo.com 


\section{Introduction}

Breast cancer remains to be a leading cause of death among women in the world. The American Cancer Society estimates that approximately 182,460 women in the US have been diagnosed with invasive breast cancer in 2009. About 40,480 women have died from this disease in this year [1]. Different methods have been used to classify and/or detect anomalies in medical images, such as wavelet transform, fractal theory, and statistical methods. In addition, some other methods were presented in the literature based on fuzzy set theory, Markov models and neural networks [2]. Computer-aided diagnostics has become promising tool for radiologists to obtain accurate and faster diagnosis result for breast cancer patients and to reduce the mortality rate [3], [4]. Most of the computer-aided methods can assist physicians and lead to better and reliable results [5].

The use of breast magnetic resonance image (MRI) has a fundamental impact on the detection and diagnosis of breast cancer [6], [7], [8]. Breast MRI demonstrates advantages over other imaging modalities. These include high resolution monitoring of high-risk patients and treatment planning, better detection and tracking of lesions [9].

The classification of data by Artificial Neural Networks (ANN) has become a widely used technique. By using examples of given tissue class specification, the neural network is capable to learn the distribution patterns of features of each class (supervised learning) [10]. Biologically inspired ANNs are robust systems come out of a highly interconnected set of processing units [11]. There are four reasons to use an artificial neural network: (i) weights can be found by iterative training, (ii) the structure of them is simple, and therefore their implementation is not difficult, (iii) complex distributions can be mapped by using ANN, and (iv) appropriate results which are non-existent in the training set can be generated for input vectors because of the generalization characteristic of the them, [12]. ANN image processing techniques take a remarkable part in the literature [13]. Most CAD algorithms based on putative classification schemes such as the K-nearest neighbour rule (KNN), Bayesian classifier and ANN [14].

The main advantage of ANN is its flexibility and learning capability from examples with linear and nonlinear data structures [15]. In the last decade, artificial intelligence (AI) has become very important in the field of medicine [16], [17], [18].

Many different learning algorithms have been developed for the training of ANNs. Some of them are Backpropagation, Delta-Bar-Delta, Fast Propagation, and Levenberg-Marquardt algorithms. But one of the most often used algorithms in ANNs is back propagation [19].

In the study presented, a system was designed by using ANN, which learns tissue types on dynamic breast MRI, and distinguishes the malignant and benign tissue types. The aim of the designed system is to assist radiologists for breast tissue diagnosis. Designed system can be trained and it can use the data learned from the training for diagnosis of malignant and benign breast tissues.

The application developed is superior to conventional methods with its ability to be trained, to learn and to adapt to new circumstances through retraining. In the application, the radiologist was provided also with the opportunity of manual MRI analysis and can see the grey values of a certain marked area both in tables 
Bayram B. et al.: An efficient algorithm for automatic tumor detection...

and in graphics. Thereby, the radiologist can interpret the areas that he or she considers suspicious or that ANN could not provide a result for

In ANN model, the backpropagation learning algorithm was adopted. As the activation function, the sigmoid activation function was used as it is one of the most appropriate ones for the backpropagation model.

Radiologically diagnosed 18 different MRIs were used for the purpose of ANN training. Of all these, 7 images consisted of MRIs with malignant tissue samples, 8 images consisted of MRIs with benign tissue samples and 3 images consisted of MRIs with normal tissue samples. The algorithm was then applied to 34 data not taken for training, and achieved a weighted accuracy of $92 \%$ when compared with the findings of the radiologists.

\section{Methodology}

Dynamic contrast-enhanced MRI of the breast is an important diagnostic tool to detect breast diseases and provides super sensitivity [7], [9], [20], [21], [22], [23], $[24]$.

The most important dynamic criterion is the relative enhancement, introduced in [6], [25]. Type I a kinetic behaviour with a constant uptake is treated a sign of benignity. Benign lesions can be extracted often in this type; Type II has a kinetic behaviour with a clear plateau phase. It can be found both in benign and in malignant features; therefore the classification of Type II curves is unknown; Type III has strong uptake behaviour. Rapid washout follows it. This behaviour is considered a sign of malignancy. Malignant lesions often have this type (Fig. 1) [6].

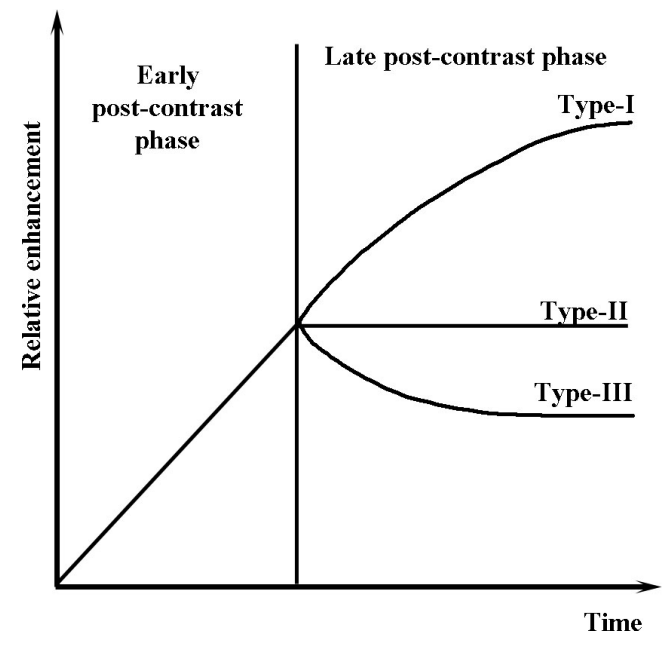

Fig. 1 Classification scheme.

In the presented study, the breast MRIs were obtained with a 1.5 - $\mathrm{T}$ system clinical scanner (Signa Advantage; IGE Medical Systems, Milwaukee, WI), with the patient prone and the uncompressed breast suspended in a proprietary IGE breast 
coil. Following axial localizer images, T2-weighted images were acquired using a FSE sequence (TR/TE of 4575/85 with frequency-selective fat suppression (SPIR; spectral presaturation inversion recovery). T2-weighted MRIs were obtained with the imaging parameters that included a slice thickness of $3 \mathrm{~mm}$, with a gap of 0.3 mm (matrix 256x224, FOV 30-48 mm).

Contrast-enhanced T1-weighted images were performed on axial imaging planes using a 3D fast low-angle shot pulse sequence (TR/TE/Flip angle-4,2/1,1/15, slice thickness of $7 \mathrm{~mm}$ without gap, matrix $384 \times 300$, FOV $30-48 \mathrm{~cm}$, TA 50s) and were obtained once before and five times after administration of $0,1 \mathrm{mmol} / \mathrm{kg}$ gadopentate dimeglumine (Magnevist-Schering). After the dynamic study, sagital T1W spin echo with fat suppression was acquired.

The application developed was essentially comprised of four main parts. These are: designing ANN through FANN (Fast Artificial Neural Network Library (http://sourceforge.net/projects/fann)), training the application, testing the training, and analysing MRIs by using the application.

FANN is a multilayer artificial neural network library for designing of ANN applications. The open-source FANN library has interfaces which enable it to be used with other programming languages.

The general characteristics of FANN Library can be summarized as follows:

1. It enables the design of a multilayer ANN,

2. It uses back propagation learning algorithm,

3. It is distributed together with the open code and LGPL ${ }^{1}$ license,

4. It can be used with .NET, $\mathrm{C}++$, Delphi, and other programming languages.

ANN has an activation function which performs the basic calculations. The activation function calculates the clear-cut outcome value (neuron sum value). The activation function is where the fundamental function of ANN is performed. Generally, it is a non-linear function.

In the developed system, the sigmoid function was adopted as the activation function. In ANNs, each connection between the neurons has a weight value which has been set randomly before learning. The weights of the connections between the neurons vary dependent on the learning as the training of ANN goes on. Besides the weight values of the connections, each neuron has a fixed value called "bias" which set randomly, as well. The summation unit is the one which calculates the "net" value of each neuron in ANN. To calculate the net value, all the input values delivered to the neuron are multiplied by the weight of connection. The fixed random value which is demonstrated as bias is added to the result [26].

$$
N E T=\left(\sum_{i=1}^{n} x(i) * w(i)\right)+\text { Bias } \quad[26]
$$

In Equation (1), $x(i) i$. refers to the input value; $w(i) i$. , the weight coefficient of the neuron connection; and bias, the fixed value set randomly.

\footnotetext{
${ }^{1}$ GNU Lesser General Public License; the free software licence issued by Free Software Foundation.
} 
Bayram B. et al.: An efficient algorithm for automatic tumor detection...

Equation (2) shows the sigmoid function result.

$$
\text { Result }=1 /\left(1+e^{-N E T}\right) \quad[26]
$$

"NET" being the value that the summation unit calculates, the output value of the neuron is calculated with the activation function. In case of ANN having more than one hidden layers, this output is entered as the input value to the neuron it is connected to in the next layer. It is essential that the error value of ANN be calculated during the first activation of ANN with the summation unit and activation function. In the first step, ANN is most likely to produce an inaccurate result. The difference between the real output and ANN's output demonstrates the error value. This value is called as $\Delta$. The error value is calculated for each neuron in the output layer. $\Delta$ is used as the apriori value in calculating the deltas of neurons in the previous layer. The $\Delta$ values of neurons in the last hidden layer are used in calculating the error values of neurons prior to it. This operation continues this way until the first hidden layer is reached. There is no error value calculation for the input layer. The calculation of connection weights by such a way in which the error value is calculated as mentioned above and given to the previous layers respectively is the back propagation learning type. In Equation (3), $\theta$ refers to the error factor, and $\rho$ refers to the expected output. Equation (3) demonstrates the way the error values of neurons in the output layer are calculated. However, it is not possible to calculate the $\theta$ value in this formula as the value relating to neurons in the hidden layers is not precisely known. Only the values of neurons in the output layer are known [26].

$$
\Delta=\text { Result } *(1-\text { result }) * \theta, \quad \theta=\rho-\text { Result } \quad[26]
$$

In the hidden layer, $\theta$ is calculated as follows:

$$
\theta_{i}=\sum_{j=0}^{n} \Delta_{j} * w_{i j} \quad[26]
$$

First the delta of each neuron which this neuron is connected to is multiplied with the weight of this connection. Summing up these delta values, the $\theta$ value of the neuron in this hidden layer is calculated. [26].

The formula in Equation (4) is demonstrated in the sample in Fig. 2.

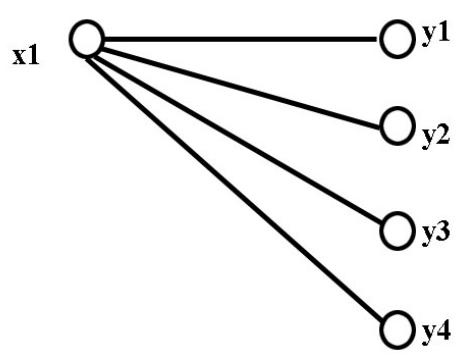

Fig. 2 The calculation of $\theta$ in hidden layer [26]. 


$$
\theta_{x 1}=\Delta_{y 1} * w_{x 1 y 1}+\Delta_{y 2} * w_{x 1 y 2}+\Delta_{y 3} * w_{x 1 y 3}+\Delta_{y 4} * w_{x 1 y 4}
$$

Once the error value calculation is over, the connection weights and the bias values set randomly as ANN was being developed are calculated again depending on these delta values. This allows the output value be more approximate to the accurate value. Following the calculation of delta and error factor, the bias value of each neuron and connection coefficients are calculated again depending on these values [26].

$$
\text { Bias }_{\text {new }}=\text { Bias }_{\text {old }}+\alpha * \Delta \quad[26]
$$

In Equation (5), $\alpha$ value refers to the learning coefficient. The learning coefficient is set between 0 and 1 . While solving basic problems, this learning coefficient is set high; however, setting the learning coefficient high in more complex problems may cause the error rate of the ANN to rise. Therefore, the learning coefficient should be decreased in more complex problems or when the error rate is high [26].

To calculate new connection weights, ANN is activated again with the new values obtained after all biases and weights are identified. ANN gets reactivated until the resulting error value reaches the desired level. If the error value does not decrease to the desired level or sticks to a certain value, there needs to be changes in the architecture of ANN. Increasing the number of hidden layers or changing the activation function or decreasing the learning coefficient are some of the changes that can be made. Besides, it is essential to use as many sample data sets as possible. (Eq. 7) [26].

$$
w_{\text {new }}=w_{\text {old }}+\alpha * \text { Result } * \Delta \quad[26]
$$

\subsection{The Design of ANN}

In this study, instead of introducing all the pixels of MRI to ANN, only one pixel or a few pixel groups were introduced to ANN as input. In this way, ANN's structure was simplified. The values obtained from each input were saved in memory, and then combined to stand as the resulting image. Dividing a big problem into small and simple parts enabled each part to contribute to the calculation of the result.

The grey value variations of pixels which share the same image coordinate were identified on dynamic MRIs. There are 8 grey value data sets for one pixel or pixel group as 8 sequential images were taken. Each of these grey value data sets was designed as an input for ANN. Therefore, the input of ANN is comprised of 8 neurons.

The grey values were categorized into 3 types depending on tissue types. These are: malignant tumor tissue, benign tumor tissue, and normal tissue. The last layer of ANN is comprised of 3 neurons as there are three different results. Each neuron corresponds to one tissue type. Tab. I shows the expected ANN results depending on the tissue type. The training data were designed so that the output values have to be 0-0-1 for the malignant tissue, 0-1-0 for the benign tissue, and 1-0-0 for the normal tissue.

Along with the input and output layers, 2 hidden layers were introduced to ANN. The number of neurons in the hidden layers was also set as 8 since this is the 
Bayram B. et al.: An efficient algorithm for automatic tumor detection...

\begin{tabular}{|c|c|c|c|c|}
\hline Tissue Type & $\begin{array}{c}\text { Output } \\
\text { Cell 1 }\end{array}$ & $\begin{array}{c}\text { Output } \\
\text { Cell 2 }\end{array}$ & $\begin{array}{c}\text { Output } \\
\text { Cell 3 }\end{array}$ & Result \\
\hline Mlignant Tissue & 0 & 0 & 1 & 001 \\
\hline Benign Tissue & 0 & 1 & 0 & 010 \\
\hline Normal Tissue & 1 & 0 & 0 & 100 \\
\hline
\end{tabular}

Tab. I ANN output according to tissue types.

number of neurons in the input layer. Furthermore, the developed system makes it possible to form as many hidden layers as needed. On the other hand, increasing the number of hidden layers for the data group in the presented study not only prolonged the process time, but also affected the result adversely. Therefore, the number of hidden layers was set as 2. As a result, ANN was designed to have 4 layers in 8-8-8-3 form; 8 of which are input layer neurons, 8 of which are first hidden layer neurons, 8 of which are second hidden layer neurons, and 3 of which are output layer neurons (Fig. 3).

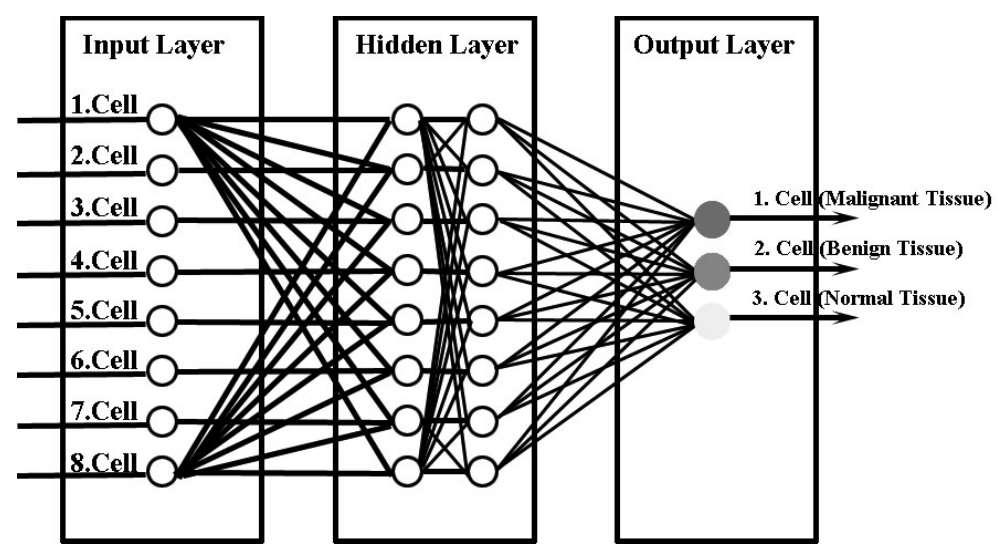

Fig. 3 Design of $A N N$.

Learning rate may be set as any value between 0 and 1 . In the presented study, 0.7 is set as the average learning value. The justification for this value is as follows: when this value is 1 , the exact value is tried to be found without any deviation, however, it is very difficult for the ANN to learn properly; If the value is low (e.g. $0.5)$, the error rate could be high due to high the deviation. Therefore, 0.7 is set as it is the average value.

\subsection{Training Data Preparation}

To stand as an example, Tab. II shows the grey value variations of the selected pixels belonging to the malignant tissue in 8 dynamic MRIs. The grey values range is from 0 to 255. Value "0" corresponds to black, and value "255" corresponds to white. The graphic for the grey value variations of pixels is shown in Fig. 4. 


\begin{tabular}{|r|rr|r|r|r|r|r|r|}
\hline MR & $\mathbf{1}$ & $\mathbf{2}$ & $\mathbf{3}$ & $\mathbf{4}$ & $\mathbf{5}$ & $\mathbf{6}$ & $\mathbf{7}$ & $\mathbf{8}$ \\
\hline $\mathbf{1}$ & 22 & 94 & 116 & 107 & 125 & 122 & 109 & 114 \\
\hline $\mathbf{2}$ & 10 & 87 & 121 & 145 & 157 & 166 & 161 & 153 \\
\hline $\mathbf{3}$ & 5 & 69 & 105 & 170 & 177 & 188 & 167 & 167 \\
\hline $\mathbf{4}$ & 6 & 54 & 72 & 87 & 98 & 98 & 120 & 123 \\
\hline $\mathbf{5}$ & 22 & 98 & 121 & 93 & 118 & 103 & 108 & 118 \\
\hline $\mathbf{6}$ & 20 & 92 & 108 & 119 & 130 & 139 & 146 & 149 \\
\hline $\mathbf{7}$ & 19 & 104 & 119 & 129 & 141 & 151 & 139 & 141 \\
\hline $\mathbf{8}$ & 10 & 94 & 109 & 109 & 109 & 117 & 112 & 115 \\
\hline $\mathbf{9}$ & 7 & 57 & 99 & 108 & 106 & 113 & 97 & 96 \\
\hline $\mathbf{1 0}$ & 4 & 36 & 69 & 87 & 116 & 134 & 121 & 122 \\
\hline $\mathbf{1 1}$ & 6 & 69 & 97 & 114 & 104 & 114 & 119 & 97 \\
\hline $\mathbf{1 2}$ & 10 & 106 & 119 & 109 & 102 & 118 & 111 & 106 \\
\hline $\mathbf{1 3}$ & 25 & 89 & 87 & 82 & 57 & 72 & 58 & 66 \\
\hline $\mathbf{1 4}$ & 5 & 92 & 104 & 118 & 120 & 130 & 130 & 119 \\
\hline
\end{tabular}

Tab. II Sample malignant tissue grey value changes.

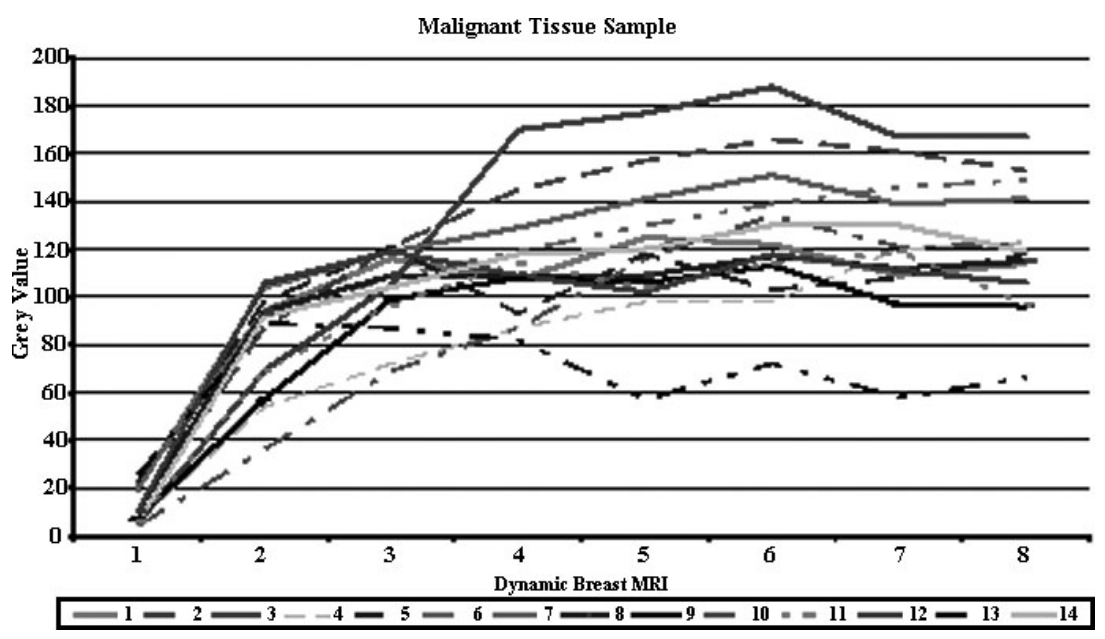

Fig. 4 Sample graph of malignant tissue grey value changes.

Although the pixels' grey value range of malignant, benign, and normal tissues taken from training data was " $0-255$ ", the values were normalized to the range 0-1 to make possible their proper introduction to ANN as an input. The grey value " 255 " was reduced to value " 1 ", and the grey value " 127 " was reduced to value "0.5".

\subsubsection{The Training of ANN}

Once, the training values are loaded, ANN training starts. The target error value and the number of training options in the developed software is identified interac- 
Bayram B. et al.: An efficient algorithm for automatic tumor detection...

tively, accompanied by how long the training will last or what the error limit will be. In the presented study, the set of number of trainings was defined as 300 , and the target training error was defined as 0,00010. According to these settings, the system tries to reach the target error 300 times (Fig. 5). If 300 trainings do not seem to be satisfactory, the number of training should be increased.

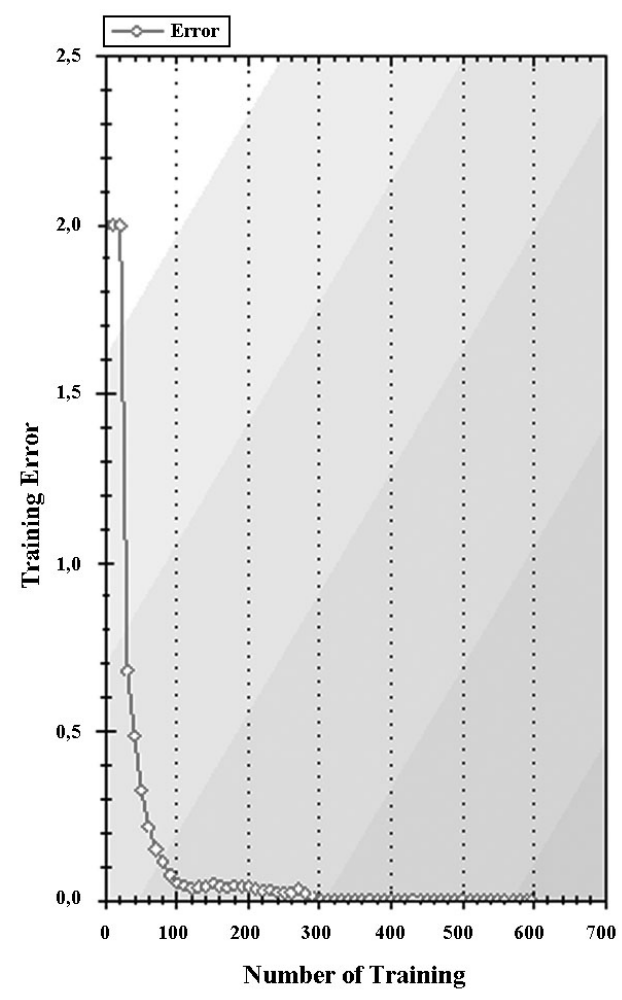

Fig. 5 Training result screen.

The developed software shows which values each output neuron met after ANN was trained and tested. Fig. 6 shows the training error graphic of ANN depending on the number of training.

Fig. 7 shows the error graphic of ANN trained with the training data set. In 84 trainings, the error value 0.0004 , which is a very good value in learning, was met.

After ANN is activated, the malignant tissue in MRI is shown in red, and the benign tissue is shown in green. The areas which tissue types could not be identified by ANN are shown in white. Fig. 8 shows the resulting image of the application. In Fig. 8, a malignant tissue accompanied by a surrounding benign tissue can be seen in the centre of the left breast. Also, in the application, the user is provided with the grey value variations of pixels in MRI, in tables and in graphics. Thereby, the user can select a specific area in MRI, and see the grey value variations of that area. This enables the physicians who use the application to obtain detailed information about the suspicious areas. 

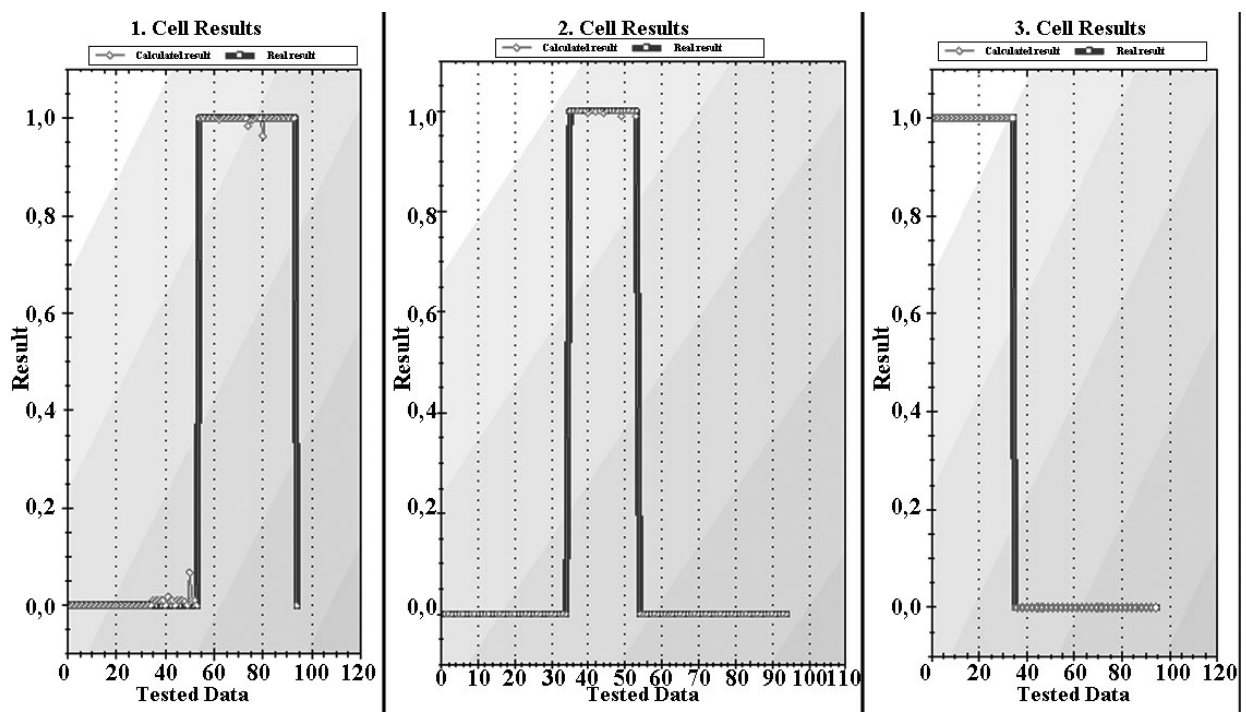

Fig. 6 Test result of sigmoid activation function.

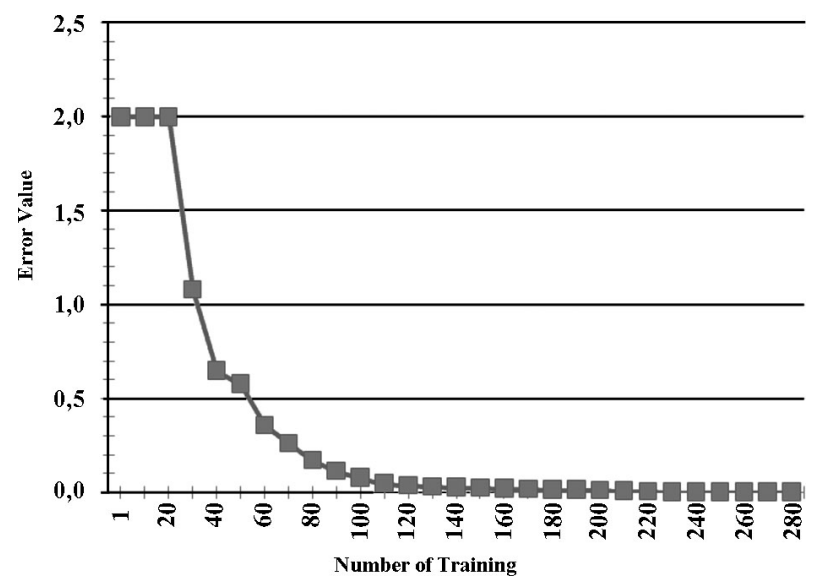

Fig. 7 Learning errors of sigmoid activation function.

The segmentation results for malignant cases are shown in Fig. 9-a, and the results for benign cases are shown in Fig. 9-b.

In the application, the pixel size can be set in order to eliminate the moving artefact effect. When the pixel size is set as " 1 ", the grey value variations of pixels sharing the same coordinate are taken. When the pixel size is set as "2", this time, the average grey value of a square area of $2 * 2=4$ pixels is calculated. This is helpful in eliminating the pixel shifts occurring in the centre of MRIs as a result of the patient's movements. In Fig. 10-a,b, the pixels on MRIs are shown. The pixel size was set as 8 to make them clear. Ideally, the pixel size should be set between 1 and 4. The pixel size was set as 8 in Fig. 10-a, and as 2 in Fig. 10-b. 
Bayram B. et al.: An efficient algorithm for automatic tumor detection...

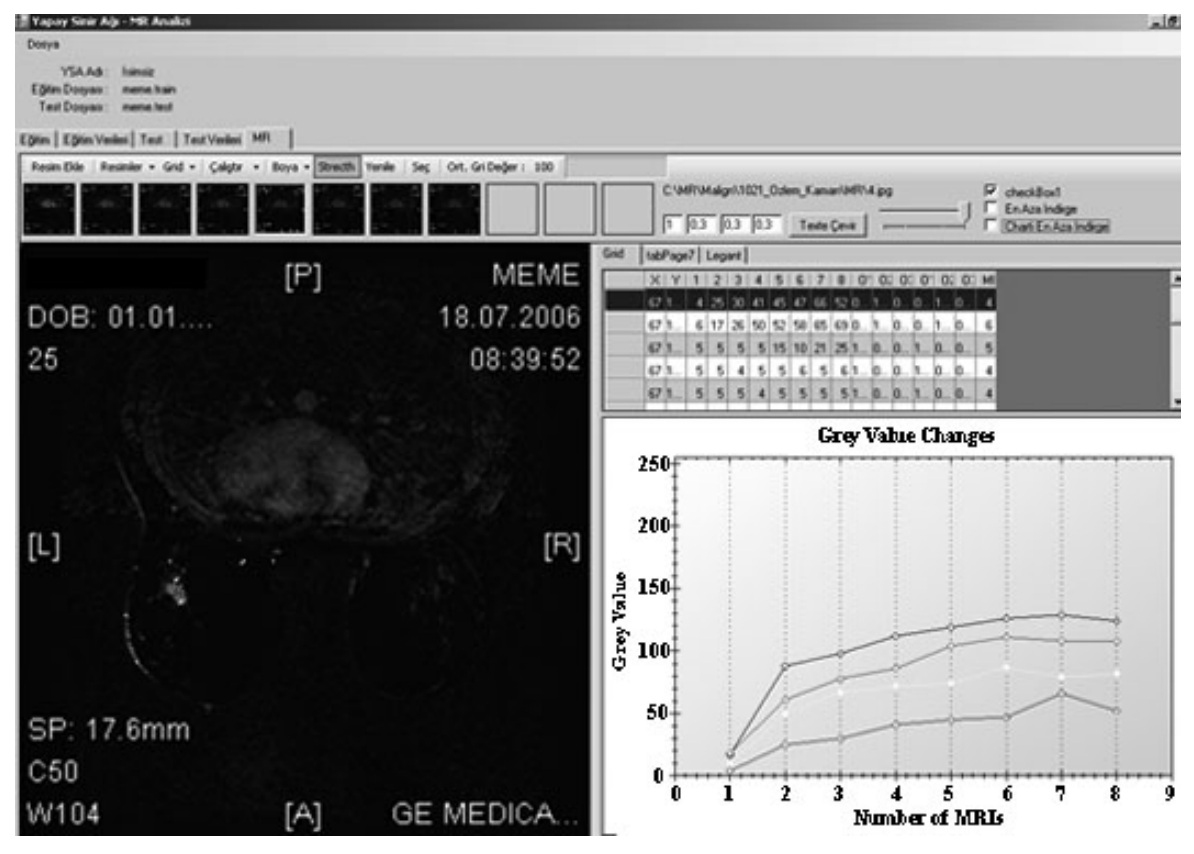

Fig. 8 Result image of the application.

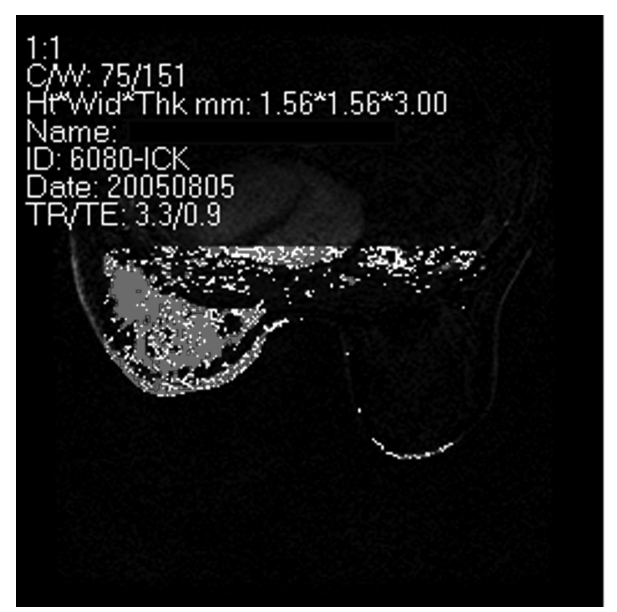

Fig. 9-a Segmentation result (malignant).

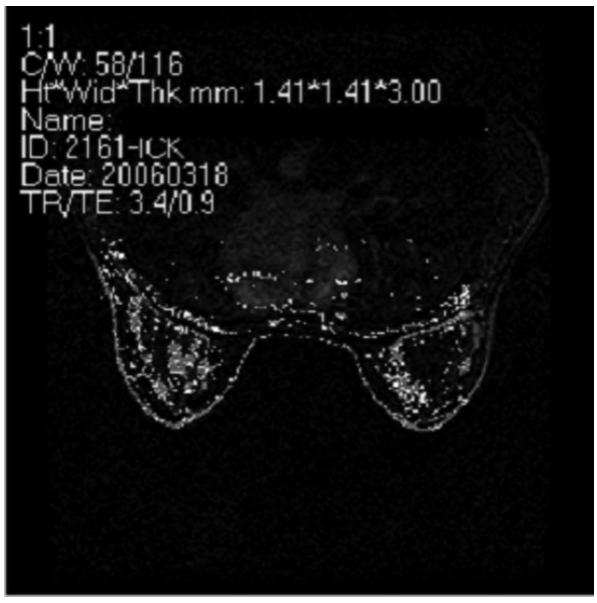

Fig. 9-b Segmentation result (benign).

\section{Results}

In the application, MRI taken from 18 cases were used as the training set. 7 of these were MRIs with malignant tissue samples, 8 of these were MRIs with benign tissue samples, and 3 of these were MRIs with normal tissue samples. The developed 


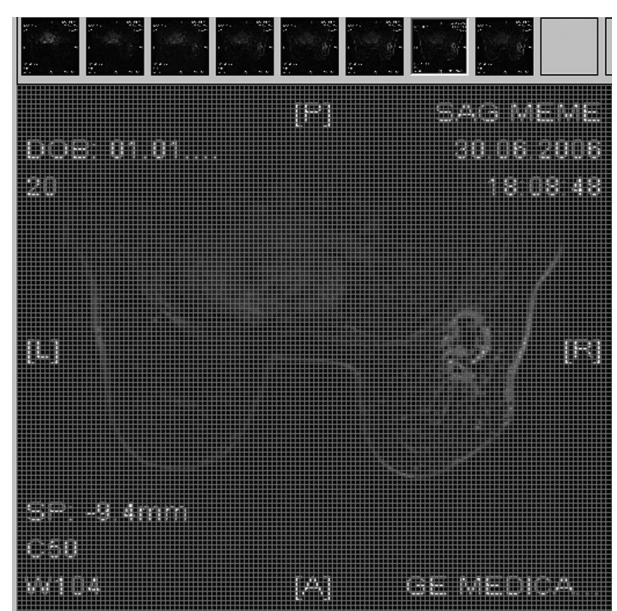

Fig. 10-a Pixel size setting as 8 .

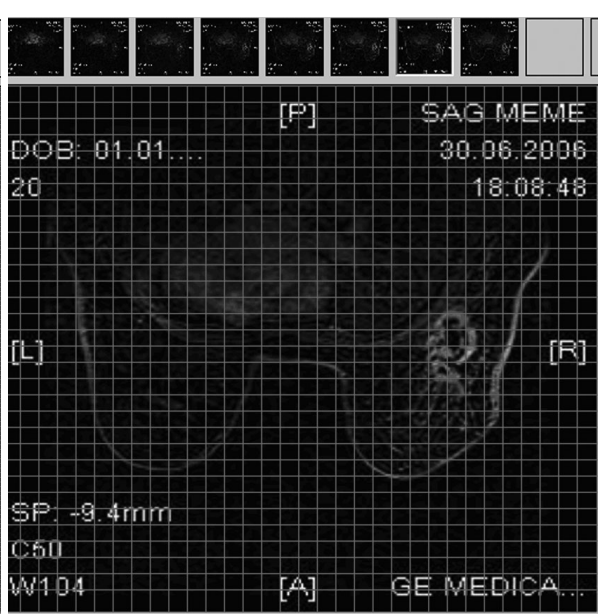

Fig. 10-b Pixel size setting as 2.

algorithm was tested for 34 cases, pre-diagnosed by radiologists, including 19 cases with benign tumor, 10 cases with malignant tumor and 5 normal cases.

10 malignant diagnosed cases have been found also malignant, 5 healthy (normal) diagnosed cases have been found healthy, 9 of 19 benign cases have been found malignant-benign, and 10 of 19 benign cases have been found benign. This result shows that the developed algorithm is malignant-sensitive. The evaluation of the results was carried out by calculating the weighted averages. Therefore, the weights of malignant-benign results were set as 0,5 and of the other results, as 1 . The result of the application was compared with the diagnosis of the radiologists. In this study, overall accuracy was $92 \%$.

In the presented study, the trained ANN can be saved and reloaded later. In this way, ANN does not need to relearn prior to each MRI analysis. Besides, the images that were used as the training set do not need to be stored. MRI is needed only during the first training.

Thanks to the "Minimize the grey values" feature, the results can be obtained regardless of the brightness values of MRIs.

The distinctive aspects of the application can be summarized as follows:

- The ability to learn from sample training data,

- Continuous learning ability: If the application produces results with errors, retraining with the obtained data enables to achieve the correct result,

- Possibility to select and analyse a certain area during MRI analysis,

- Possibility to set the pixel size in MRI analysis,

- Thanks to the "Minimize the grey values" feature, the contrast differences on MRI not causing a problem,

- Developed system provides the physician with the dynamic images of grey value variations of certain areas on MRI, 
Bayram B. et al.: An efficient algorithm for automatic tumor detection...

- Possibility to save the results of the application and use them again later,

- Ability to learn vascular tissues, adipose tissues, muscle tissues, etc., if trained with sample data, as an addition to presented malignant, benign, and normal tissue analysis.

\section{Discussion}

The most important factor in ANNs is the need for the input data to be in accordance with the data desired to be obtained. The input values you may introduce to ANN to solve a non-linear problem can vary even within the same problem. Important is to be able to reach the correct result with the most effective data as fast as possible.

In the input layer of ANN, there should be a neuron which corresponds to each element of the row in which the grey values of the image are arranged. The number of neurons in the input layer should be equal to the size of the row. For example, for a picture of 30 pixel width and 20 pixel heights, there should be $30 * 20=600$ neurons in the input layer. Similarly, there are 600 neurons in the input layer of ANN. The number of neurons in the hidden layer can be 1200, 600, 300, etc. However, if the number of the neurons in the hidden layer is reduced, for example to 100 , which is $1 / 6$ of the ones in the input layer, this will alleviate ANN's ability to learn and reach the correct result. Therefore, the number of neurons in the hidden layer should be at least the half of the ones in the input layer. Given that there were 300 neurons in the hidden layer, the total connection number for this ANN would be $600 * 300+300 * 2=180.600$.

For an MRI which has $300 * 300$ pixels the number of connections is 8.100.180.000 ( $\sim 8$ billion). $300 * 300$ pixels equal to 90.000 neurons in the input layer. In total, this equals to $8.100 .000 .000+90.000 * 2=8.100 .180 .000(\sim 8$ billion $)$ connections. It was a need to determine a numeral value of at least 8 bytes for each connection ${ }^{2}$. In this case, the required memory would be at least $8.100 .180 .000 * 8=64.801 .440 .000$ Bytes (approximately 64GB).

\begin{tabular}{|r|r|r|r|}
\hline $\begin{array}{r}\text { Image } \\
\text { Size }\end{array}$ & $\begin{array}{r}\text { Toplam } \\
\text { Boyut }\end{array}$ & $\begin{array}{r}\text { Avarege Number } \\
\text { of Connections }\end{array}$ & $\begin{array}{r}\text { Required } \\
\text { memory }\end{array}$ \\
\hline $20 * 30$ & 600 & 360.000 & $3 \mathrm{MB}$ \\
\hline $30 * 30$ & 900 & 810.000 & $6 \mathrm{MB}$ \\
\hline $50 * 50$ & 2.500 & 6.250 .000 & $48 \mathrm{MB}$ \\
\hline $100 * 100$ & 10.000 & 100.000 .000 & $763 \mathrm{MB}$ \\
\hline $150 * 150$ & 22.500 & 506.250 .000 & $3862 \mathrm{MB}$ \\
\hline $300 * 300$ & 90.000 & 8.100 .000 .000 & $64000 \mathrm{MB}$ \\
\hline $500 * 500$ & 250.000 & 62.500 .000 .000 & $476800 \mathrm{MB}$ \\
\hline
\end{tabular}

Tab. III Memory needed depending on image sizes.

\footnotetext{
${ }^{2}$ Decimal numbers need a memory field of 8 bytes
} 
According to this result (Tab. III), creation of such an ANN and training of it is not efficient for conventional desktop computers. Parallel computer techniques or super-computers can be more functional for such kind of system. In the presented study, this method was not chosen due of limitations.

In the presented study, a comparison of the sigmoid function and the linear function was made. The purpose was to determine the impact of using a different activation function on the result of learning. It was observed that the learning error was fixed on value 0,3 when ANN was trained by using linear function (Fig. 11). On the other hand, when the sigmoid function was used, the error value of 0.0004 was obtained, which is a very good value in 84 . epoch (See Fig. 7).

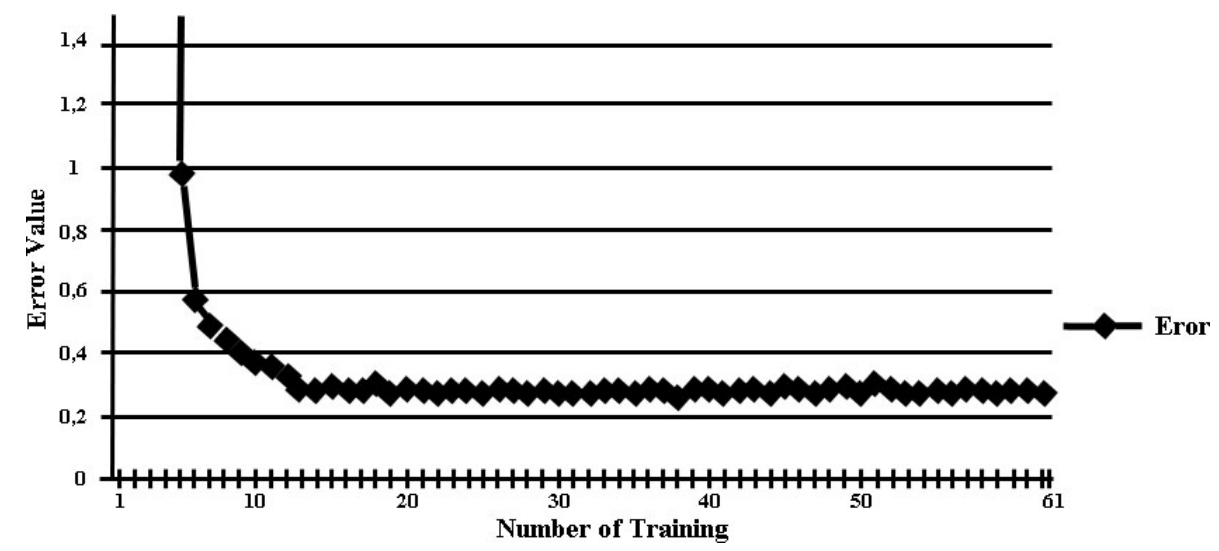

Fig. 11 Training errors of linear activation function.

Fig. 12 shows the test results of linear activation function. As it can be seen in Fig. 12, when a linear function is used, ANN cannot obtain the desired result. Therefore, linear activation function was not used.
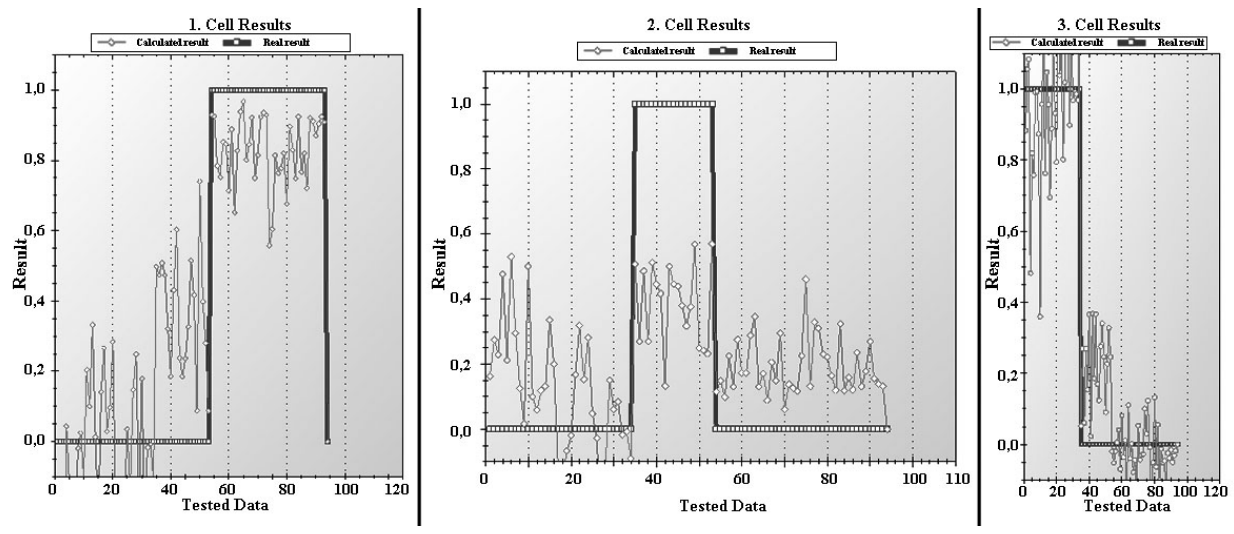

Fig. 12 Linear activation function results. 
Bayram B. et al.: An efficient algorithm for automatic tumor detection...

The developed method should never be used without the supervision of a physician. This study was not developed as an intelligent system; it has been developed only to assist physicians for diagnosis.

Breast MRIs were used in the presented study. However, in terms of its function, the developed system is not dependent on the cancer type to be examined. This system can be used for analysis of different tissues and organs as well. For example, the developed system is able to be trained with the grey value variations of vascular tissue type. It is possible sometimes to mix vascular tissue with tumor tissue. By using developed system, vascular tissue can be distinguished from tumor tissue to avoid such kind of errors. These errors can be examined and distinguished by the physician as the application provides the user with the grey value variation graphic of a certain area.

Physicians perform morphological analysis along with radiometric analysis during diagnosis. Thus, the studies on enabling the algorithm to perform morphological analysis are in progress. Thereafter, 3D tumor modelling and creation of virtual reality tumor model for simulation of the surgery has been planned as next step of this study.

\section{References}

[1] Liyang Wei, Yongyi Yang, Robert M. Nishikawa,: Microcalcification classification assisted by content-based image retrieval for breast cancer diagnosis, Pattern Recognition, 42, 2009, pp. 1126-1132.

[2] Ferrero, G., Britos, P., García-Martínez, R.: Detection of Breast Lesions in Medical Digital Imaging Using Neural Networks. IFIP International Federation for Information Processing, Professional Practice in Artificial Intelligence, eds. J. Debenham, (Boston: Springer), Volume 218, 2006, pp. 1-10.

[3] Stoitsis J., Valavanis I., Mougiakakou S. G., Golemati S., Nikita A., Nikita K. S.: Computer aided diagnosis based on medical image processing and artificial intelligence methods, Nuclear Instruments and Methods in Physics Research, vol. 569, 2006, pp. 591-595.

[4] Esugasini Subramaniam, Tan Kuan Liung, Mohd. Yusoff Mashor, Nor Ashidi Mat Isa: Breast Cancer Diagnosis Systems: A Review, International Journal of The Computer, the Internet and Management, Vol. 14., No. 2, 2006, pp. 24-35.

[5] Antonie M., Zaïene O., Coman A.: Application of data mining techniques for medical image classification, Proceedings of the Second International Workshop on Multimedia Data Mining. San Francisco, 2001, pp. 94-101.

[6] Cardillo F. A., Starita A., Caramella D., Cilotti A., Odoguardi F.: A simple comparison of dynamic criteria for breast MRI classification, Proceedings of the European Conference on Emergent Aspects in Clinical Data Analysis (EACDA 05). September 28-30, Pisa, Italy, 2005, pp. 103-110.

[7] Takeda Y., Yoshikawa K.: Contrast-enhanced dynamic MR imaging parameters and histological types of invasive ductal carcinoma of breast, Biomedicine \& Pharmacotherapy, vol. 59, 2005, pp. 115-121.

[8] Behrens S., Laue H., Althaus M., Boehler T., Kuemmerlen B.: Computer assistance for MR based diagnosis of breast cancer: Present and future challenges, Computerized Medical Imaging and Graphics, 31, 2007, pp. 236-247.

[9] Xiaobing Fan, Milica Medved, Gregory S. Karczmar, Cheng Yang, Sean Foxley, Sanaz Arkani, Wendy Recant, Marta A. Zamora, Hiroyuki Abe, Gillian M. Newstead: Diagnosis of suspicious breast lesions using an empirical mathematical model for dynamic contrast-enhanced MRI, Magnetic Resonance Imaging. Jun. 25, vol. 5, 2007, pp. 593-603. 


\section{Neural Network World 5/13, 483-498}

[10] Lucht R. E. A., Knopp M. V., Brix G.: Classification of signal-time curves from dynamic MR mammography by neural Networks, Magnetic Resonance Imaging, vol. 19, 2001, pp. $51-57$.

[11] Szabó B., Wiberg M., Boné B., Aspelin P.: Application of artificial neural networks to the analysis of dynamic MR imaging features of the breast, European Radiology, Volume 14, Number 7, 2004, pp. 1217-1225.

[12] Zümray Dokur: A unified framework for image compression and segmentation by using an incremental neural network, Expert Systems with Applications, vol. 34, 2008, pp. 611-619.

[13] Giuseppe Coppini, Stefano Diciotti, Massimo Falchini, Natale Villari, and Guido Vali: Neural Networks for Computer-Aided Diagnosis: Detection of Lung Nodules in Chest Radiograms, IEEE Transactions On Information Technology In Biomedicine, Vol. 7, No. 4., 2003, pp. 344-357.

[14] M. Park, B. Kang, S. J. Jin, S. Luo: Computer aided diagnosis system of medical images using incremental learning method, Expert Systems with Applications, vol. 36, 2009, pp. $7242-7251$.

[15] Marcel Volmer, Bert G. Woithers, Harm J. Metting, Thus H. Y. de Haan, Pierre M. J. Coenegracht, and Wim van der Slik: Artificial Neural Network Predictions of Urinary Calculus Compositions Analyzed with Infrared Spectroscopy, Clinical Chemistry, 40/9, 1994, pp.1692-1697.

[16] Antonio Ciampi and Fulin Zhang: A new approach to training back-propagation artifcial neural networks: empirical evaluation on ten data sets from clinical studies, Statistics In Medicine, vol. 21, 2002, pp. 1309-1330.

[17] Fatih Kurugollu, Bülent Sankur, A. Emre Harmancı: Image segmentation by relaxation using constraint satisfaction neural network, Image and Vision Computing, vol: 20, 2002, pp. 483-497.

[18] Paulo J. Lisboa, Azzam F. G. Taktak: The use of artificial neural networks in decision support in cancer: A systematic review, Neural Networks, vol. 19, 2006, pp. 408-415.

[19] Haykin S.: Neural Networks, A Comprehensive Foundation, Prentice Hall International, ISBN: 0-13-908385-5, 1999.

[20] Harms S. E., Flamig D. P.: MR imaging of the breast: technical approach and clinical experience. Radiographics, vol. 13, 1993, pp. 905-12.

[21] Heywang-Kobrunner SH, Viehweg P, Heinig A, Kuchler C. : Contrastenhanced MRI of the breast: accuracy, value, controversies, solutions. Eur J Radiol., vol. 24, 1997, pp. 94-108.

[22] Kuhl C. K., Mielcareck P., Klaschik S.: Dynamic breast MR imaging: are signal intensity time course data useful for differential diagnosis of enhancing lesions? Radiology, vol. 211, 1999, pp. 101-110.

[23] Kriege M., Brekelmans C. T., Boetes C., et al. : Efficacy of MRI and mammography for breast-cancer screening in women with a familial or genetic predisposition. N Engl J Med. Vol., 351, 2004, pp. 427-437.

[24] Kurz K. D., Steinhaus D., Klar V., Cohnen M., Wittsack H. J., Saleh A., Mödder U., Blondin D.: Assessment of three different software systems in the evaluation of dynamic MRI of the breast, European Journal of Radiology, vol. 69, 2009, pp. 300-307.

[25] Kaiser W. A., Zeitler E.: MR imaging of the breast: fast imaging sequences with and without Gd-DTPA. Preliminary observations, Radiology, Part: Part 1, vol. 170, issue 3, 1989, pp. 681-686.

[26] K. Koca, Hilmi: Automatic Tumor Segmentation In Contrast-Enhanced Dynamic MRI By Using Artificial Neural Network Technique, Msc Thesis, Yildiz Technical University, Institute of Natural and Applied Science, 2008. 